\title{
Barotropic instabilities of the Agulhas Current system and their relation to ring formation
}

\author{
Henk A. Dijkstra and Wilhelmus P. M. de Ruijter \\ Institute for Marine and Atmospheric research Utrecht \\ Utrecht University \\ Utrecht, the Netherlands
}

Submitted to J. Marine Research

Version of February 5, 2001

Corresponding Author:

Henk A. Dijkstra

Institute for Marine and Atmospheric research Utrecht

Department of Physics and Astronomy

Utrecht University

Princetonplein 5, 3584 CC Utrecht

The Netherlands

Phone: -31-30-2533858; Fax: -31-30-2543163

Email: dijkstra@phys.uu.nl 


\begin{abstract}
Recent work has shown that different steady retroflection regimes, a viscous and an inertial regime, exist for the Agulhas Current system in an idealized geometry. In this paper, instabilities of these steady flows are considered by solving the linear stability problem numerically. Barotropic instabilities occur as so-called Hopf bifurcations in the viscous regime, with corresponding patterns related to Rossby basin modes. Depending on the value of the reduced gravity parameter, these instabilities may introduce intermonthly to interannual variability into the retroflection region. Finite amplitude development of these instabilities display 'ring-like' localized anomaly patterns which travel around the tip of the continent. The results demonstrate that (i) the origin of the frequency of the ring formation is set by the physics of the large scale barotropic instabilities and (ii) the rectification processes due to these instabilities decrease the degree of retroflection of the mean state. The latter result suggests that the dominant mechanism of the retroflection is captured within the steady balances.
\end{abstract}




\section{Introduction}

Roughly once every two months, a large ring is shed from the Agulhas Current and propagates westward into the South Atlantic (Gordon and Haxby, 1990; Feron et al., 1992; Schouten et al., 2000). The Agulhas Current itself turns eastward in an impressive retroflection, leaving the rings as main process of interbasin exchange. As the rings transport heat and salt from the Indian to the Atlantic Ocean, they are potentially important for the global thermohaline circulation (Gordon, 1985; De Ruijter et al., 1999). Estimates on the amount of mass, heat and salt involved in the exchange differ widely, both from observations and from different general circulation models (De Ruijter et al., 1999).

In a detailed numerical model study (Chassignet and Boudra, 1988), ring formation is considered in an isopycnic (and in most cases two-layer) model for an idealized domain under a wide range of conditions. In the relatively viscous regime (the low Rossby number case E1), 4 rings per year are found on average. The frequency of ring cut-off was shown to be related to the interaction of the top-layer flow dynamics and ocean basinmode patterns propagating in the bottom layer. Energy transformations in the flow were monitored but did not give any conclusive evidence whether a type of instability plays a primary role in the ring formation and shedding process. In the more inertial regime (the large Rossby number case E10), the frequency decreases to 4 rings in 10 years. In this case, a strong coupling exists between the ring shedding and the retroflecting and meandering mean current. During a ring shedding event, the bottom layer kinetic energy triples in magnitude and a mixed barotropic-baroclinic instability was suggested to be involved. Processes setting the frequency of the ring shedding events were in this case difficult to identify.

A pure inertial mechanism of ring formation has been proposed recently in the context of a reduced gravity model (Nof and Pichevin, 1996; Pichevin et al., 1999). 
When a current retroflects, a momentum imbalance exists under a rather restricting set of conditions. Development of rings can solve this so-called "retroflection paradox" and the theory developed provides estimates of spatial scales of the rings and their propagation speed. Numerical results show reasonable agreement with the theoretically provided bounds on length scale and mass flux of the rings. Only the period of ring shedding appears slightly more sensitive to parameter changes and varies within a range of 80 - 160 days. The existence of these different views of ring formation is intriguing and their connection is not easily established; model formulations and corresponding dynamics are very different.

In an earlier study (Dijkstra and De Ruijter, 2000), we reconsidered the small basin configuration (De Ruijter and Boudra, 1985; Chassignet and Boudra, 1988) and analysed the steady states within a barotropic shallow water model as a function of the lateral friction coefficient (horizontal Ekman number E). Using modern tools of numerical bifurcation theory, also unstable steady states could be determined and two regimes of steady retroflection were shown to exist, a viscous and an inertial one. In the viscous regime, retroflection occurs through viscous choking; the viscous boundary layer is thick enough to allow the current to bridge the gap from the southern tip of the continent to the latitude of zero wind stress curl. In the inertial regime, inertial effects are strong enough to bridge this gap (De Ruijter and Boudra, 1985). Most important result in Dijkstra and De Ruijter (2000) is that retroflection characteristics are already present in the steady balances and that rectification processes due to barotropic/baroclinic instabilities are not essential for retroflection to occur.

In this paper, we consider the stability of these steady solutions by solving the linear stability problem numerically within an (equivalent) barotropic shallow water model. The different unstable modes are identified and their characteristics are studied when varying the Rossby deformation radius. For the barotropic case, transient flows in the unstable regime are computed and the physics of the dominant time scale of variability 
and the changes in the time mean state are investigated.

\section{Model and methods}

The model used (Dijkstra and De Ruijter, 2000) is a (equivalent) barotropic shallow water model in spherical coordinates, modelling the zonal velocity $u$, meridional velocity $v$ and sea surface height anomaly $h$ (with respect to some equilibrium depth $H$ ) of the wind-driven flow in the upper layer having density $\rho$. Below this layer is a motionless layer with density $\Delta \rho+\rho$, such that the reduced gravity $g^{\prime}=g \Delta \rho / \rho$. In this paper, we will use only the idealized basin geometry as in De Ruijter and Boudra (1985). The domain chosen is $\phi_{W}=10^{\circ} \mathrm{E}, \phi_{E}=32^{\circ} \mathrm{E}, \theta_{S}=42^{\circ} \mathrm{S}$ and $\theta_{N}=30^{\circ} \mathrm{S}$, and the basin has a flat bottom. The zonal size of the domain varies with latitude (from about $2100 \mathrm{~km}$ at the northern boundary to about $1800 \mathrm{~km}$ at the southern boundary) and has about the same dimensions as in De Ruijter and Boudra (1985), with a meridional extent of $1300 \mathrm{~km}$. A 'rectangular' continent is present over the domain $\phi_{W}=19.5^{\circ} \mathrm{E}$, $\phi_{E}=20.5^{\circ} \mathrm{E}, \theta_{S}=35^{\circ} \mathrm{S}$ and $\theta_{N}=30^{\circ} \mathrm{S}$.

The wind stress forcing is prescribed as an analytical profile of the form

$$
\tau^{\phi}(\theta)=\cos \pi\left(6.37\left(\theta-\theta_{*}\right)\right) ; \tau^{\theta}=0
$$

and $\tau^{\phi}(\theta)$ is plotted in Fig. 1 for $\theta_{*}=-39.5$. The amplitude $\tau_{0}$ was chosen similar to that in De Ruijter and Boudra (1985), i.e. $\tau_{0}=0.2 \mathrm{~Pa}$. In this way, the maximum westerly wind stress is located at $\theta=-39.5$, i.e. about $400 \mathrm{~km}$ south of the tip of the continent. Although the wind stress curl is not exactly zero at this location (because of the spherical coordinates), the deviation is small because of the small basin size.

With $r_{0}$ denoting the radius of the earth, the governing shallow water equations are non-dimensionalized using scales $r_{0}, H, U, r_{0} / U$ and $\tau_{0}$ for length, layer depth, velocity, 
time and wind stress, respectively. These become

$$
\begin{array}{r}
\epsilon\left(\frac{\partial u}{\partial t}+\frac{u}{\cos \theta} \frac{\partial u}{\partial \phi}+v \frac{\partial u}{\partial \theta}-u v \tan \theta\right) \\
-v \sin \theta=-\frac{\epsilon F}{\cos \theta} \frac{\partial h}{\partial \phi}+E\left(\nabla^{2} u-\frac{u}{\cos ^{2} \theta}-\frac{2 \sin \theta}{\cos ^{2} \theta} \frac{\partial v}{\partial \phi}\right)+\alpha \frac{\tau^{\phi}}{h} \\
\epsilon\left(\frac{\partial v}{\partial t}+\frac{u}{\cos \theta} \frac{\partial v}{\partial \phi}+v \frac{\partial v}{\partial \theta}+u^{2} \tan \theta\right) \\
+u \sin \theta=-\epsilon F \frac{\partial h}{\partial \theta}+E\left(\nabla^{2} v-\frac{v}{\cos ^{2} \theta}+\frac{2 \sin \theta}{\cos ^{2} \theta} \frac{\partial u}{\partial \phi}\right)+\alpha \frac{\tau^{\theta}}{h} \\
\frac{\partial h}{\partial t}+\frac{1}{\cos \theta}\left(\frac{\partial(h u)}{\partial \phi}+\frac{\partial(h v \cos \theta)}{\partial \theta}\right)=0
\end{array}
$$

In the dimensionless equations, the following parameters appear, i.e. the Rossby number $\epsilon$, the rotational Froude number $F$, the Ekman number $E$, and the wind stress coefficient $\alpha$. Expressions for these parameters are

$$
\epsilon=\frac{U}{2 \Omega r_{0}} ; F=\frac{g^{\prime} H}{U^{2}} ; E=\frac{A_{H}}{2 \Omega r_{0}^{2}} ; \alpha=\frac{\tau_{0}}{2 \Omega \rho H U}
$$

where $\Omega$ is the angular velocity of the earth. Standard values of both dimensional and non-dimensional parameters are given in Table 1 and are similar as in Dijkstra and De Ruijter (2000). The only difference is the value of $F$, which in the barotropic limit is equal to $10^{6}$, the value in the limit $g^{\prime} \rightarrow g$.

Branches of the steady states are followed in parameter space, mainly in the control parameter $E$, using continuation techniques. For each solution, the retroflection index $\mathcal{R}$, as defined in Dijkstra and De Ruijter (2000), is plotted. This retroflection index is the ratio of the maximum westward volume transport $\left(\Phi_{w}(20)\right)$ at the tip of the continent $\left(20^{\circ} \mathrm{E}\right)$ and the maximum southward volume transport $\left(\Phi_{s}(-35)\right)$ at $35^{\circ} \mathrm{S}$. In the results below, $\mathcal{R}$ is computed as

$$
\mathcal{R}=1-\frac{\Phi_{w}(20)}{\Phi_{s}(-35)}
$$

If there is no westward transport $\left(\Phi_{w}(20)=0\right)$, then there is complete retroflection and $\mathcal{R}=1$. On the other hand, if all the incoming southward flow from the Agulhas Current 
is transported westward, then there is no retroflection at all and $\mathcal{R}=0$. The results in Dijkstra and De Ruijter (2000) indicate that for solutions computed on $1 / 4^{\circ}$ (as is the resolution for most results in De Ruijter and Boudra (1985)), the Ekman number should be larger than about $E=2 \times 10^{-7}$.

The equations (2) can be written in operator form as

$$
\mathcal{M} \frac{\partial \mathbf{u}}{\partial t}+\mathcal{L} \mathbf{u}+\mathcal{N}(\mathbf{u})=\mathbf{F}
$$

where $\mathcal{L}, \mathcal{M}$ are linear operators, $\mathcal{N}$ is a nonlinear operator, $\mathbf{u}$ is the vector of dependent quantities and $\mathbf{F}$ contains the forcing of the system and appropriate boundary conditions are taken into account. To determine the steady states $\overline{\mathbf{u}}$ of the system of equations when parameters are changed, the problem

$$
\mathcal{L} \overline{\mathbf{u}}+\mathcal{N}(\overline{\mathbf{u}})=\mathbf{F}
$$

has to be solved. This set of partial differential equations is discretized by a central volume finite difference method on a $88 \times 48$ grid, having a horizontal resolution of $1 / 4^{\circ}$, and a system of nonlinear algebraic equations of the form

$$
\mathbf{G}(\mathbf{x}, \mu)=0
$$

emerges. Here $\mathbf{x}$ is a $d=3 \times 88 \times 48=12,672$-dimensional vector consisting of the unknowns at the gridpoints, $\mu$ is the control parameter, for example $\mu=E$, and $\mathbf{G}$ is a nonlinear mapping from $R^{d} \times R \rightarrow R^{d}$. To determine branches of steady solutions of (7) as $\mu$ is varied, the pseudo-arclength method is used (Keller, 1977; Seydel, 1994). The branches $(\mathbf{x}(s), \mu(s))$ are parametrized by an 'arclength' parameter $s$ and an additional equation is obtained by an approximation of the normalization condition of the tangent, i.e.

$$
\dot{\mathbf{x}}_{0}^{T}\left(\mathbf{x}-\mathbf{x}_{0}\right)+\dot{\mu}_{0}\left(\mu-\mu_{0}\right)-\Delta s=0
$$

where $\left(\mathbf{x}_{0}, \mu_{0}\right)$ is a known starting solution or a previously computed point on a particular branch, $\Delta s$ is the steplength and the dot indicates differentiation to $s$. To follow 
steady solutions along branches in parameter space, an Euler-Newton scheme is used (Seydel, 1994).

To determine the linear stability of a steady state, perturbations $\tilde{\mathbf{u}}$ are considered. With $\mathbf{u}=\overline{\mathbf{u}}+\tilde{\mathbf{u}}$, linearizing (5) around $\overline{\mathbf{u}}$ and separating $\tilde{\mathbf{u}}=\hat{\mathbf{u}} e^{\sigma t}$ gives an eigenvalue problem of the form

$$
\left(\mathcal{L}+\mathcal{N}_{u}\right) \hat{\mathbf{u}}=-\sigma \mathcal{M} \hat{\mathbf{u}}
$$

where $\mathcal{N}_{u}$ is the derivative of the operator $\mathcal{N}$ with respect to $\mathbf{u}$. Discretization of this eigenvalue problem leads to a generalized algebraic eigenvalue problem of the form

$$
\mathcal{A} \hat{\mathbf{x}}=\sigma \mathcal{B} \hat{\mathbf{x}}
$$

where $\mathcal{A}$ is a non-singular, non-symmetric $d \times d$ matrix and $\sigma=\sigma_{r}+i \sigma_{i}$ is the eigenvalue. The problem (10) is solved by the Jacobi-Davidson QZ-method (JDQZ). With this method, one can compute several eigenvalues and optionally eigenvectors near a specified target. Details of the method are described in Sleijpen and Van der Vorst (1996) and the implementation of JDQZ in an earlier version of our continuation code in Van Dorsselaer (1997).

\section{Results: Stability bounds}

In this section, first the linear stability of the barotropic steady states computed in Dijkstra and De Ruijter (2000) is discussed and subsequently effects of changes in the Rossby deformation radius are studied.

As mentioned above, the barotropic (flat bottom) case can be obtained in the limit $g^{\prime} \rightarrow g$ giving the value $F=10^{6}$. Two bifurcation diagrams are combined in Fig. 2 for two values of the layer thickness $H$. The curves are similar to Fig. 5 in Dijkstra and De Ruijter (2000), except that now the stability of the steady flows is indicated. A solid (dotted) curve indicates a stable (unstable) flow, while the stability boundaries are 
marked by the solid triangles. Here, the steady states becomes unstable to oscillatory instabilities; such a transition is called a Hopf bifurcation (Seydel, 1994). For $H=$ $1000 \mathrm{~m}$, this stability boundary is located at $E_{c}=2.6 \times 10^{-7}$ whereas for $H=250 \mathrm{~m}$, it is found at $E_{c}=8.2 \times 10^{-7}$. As could be expected, the case with higher inertially controlled flow is more unstable, because of the larger horizontal shear.

At the Hopf bifurcation on the curve for $H=1000 \mathrm{~m}$ in Fig. 2, the steady state flow pattern, shown as a contour plot of the layer thickness anomaly $h$, is plotted in Fig. 3a. A strong Agulhas Current is present near the eastern side of the continent but it has almost no retroflection. The pattern of the oscillatory mode which destabilizes the steady state can be determined from the solution of the linear stability problem (10) with eigenvalue $\sigma$. The corresponding complex eigenfunction $\hat{\mathbf{x}}=\hat{\mathbf{x}}_{R}+i \hat{\mathbf{x}}_{I}$ provides the time periodic disturbance structure $\mathbf{P}(t)$ with angular frequency $\sigma_{i}$ and growth rate $\sigma_{r}$ to which the steady state becomes unstable, i.e.

$$
\mathbf{P}(t)=e^{\sigma_{r} t}\left[\hat{\mathbf{x}}_{R} \cos \left(\sigma_{i} t\right)-\hat{\mathbf{x}}_{I} \sin \left(\sigma_{i} t\right)\right]
$$

Propagation features of a neutral mode $\left(\sigma_{r}=0.0\right)$ can be determined by first looking at $\mathbf{P}\left(-\pi /\left(2 \sigma_{i}\right)\right)=\hat{\mathbf{x}}_{I}$ and then at $\mathbf{P}(0)=\hat{\mathbf{x}}_{R}$. The dimensional period $\mathcal{P}$ of the oscillation is given by $\mathcal{P}=2 r_{0} \pi /\left(U \sigma_{i}\right)$.

The patterns of the real and imaginary part of the eigenvector, destabilizing the steady state in Fig. 3a are shown in Fig. 3b-c. The dimensionless angular frequency of the mode $\left(\sigma_{i}=1.12 \times 10^{2}\right)$ corresponds to a dimensional period $\mathcal{P}=1.35$ months. The perturbation patterns are basin wide and have strong similarities to inviscid Rossby basin modes (Pedlosky, 1987), which can be labelled $(n, m)$ according to the number of zeroes of the streamfunction in zonal and meridional direction. The patterns here would be 'guessed' as the $(2,1)$ mode which in the inviscid case has an oscillation period of about a month (Chassignet and Boudra, 1988; Dijkstra and Katsman, 1997). Hence, the pattern in Fig. 3b-c can be identified as a destabilized Rossby basin mode. In this 
case, the presence of the continent deforms the basin mode as it propagates westwards. The steady flow with smaller layer thickness $(H=250 \mathrm{~m})$ destabilizes already at larger values of the lateral friction. The steady state (Fig. 4a) at criticality $\left(E_{c}=\right.$ $8.2 \times 10^{-7}$ ) shows already some more tendency to retroflection, although from Fig. 2, it can be seen that steady retroflection becomes only pronounced at smaller values of E. The patterns of the most unstable mode at Hopf bifurcation (Fig. 4b-c) resemble again a westward propagating Rossby basin mode ('guessed' as the pattern coming from the inviscid $(4,1)$ Rossby basin-mode) and its oscillating period is about 1.4 months $\left(\sigma_{i}=1.09 \times 10^{2}\right)$. The main difference between this mode and the mode in Fig. 3 (in the case $H=1000 \mathrm{~m}$ ) is that the pattern is more localized within the high shear region of the steady state and has a smaller zonal scale.

For the case $H=1000 \mathrm{~m}$, the values of the lateral Ekman number $E$ at which the Hopf bifurcation occurs (and the corresponding angular frequency of oscillation at criticality) are plotted in Fig. 5 for several values of $F$. The frequency of oscillation (dashed curve) is increasing monotonically with $g^{\prime}$ as is expected from elementary Rossby wave theory. For $F=1000$, the value of $\sigma_{i}$ has decreased to $\sigma_{i}=7.8$ which corresponds to an oscillation period of about 1.6 years. The critical value $E_{c}$ has no monotonic behavior and there seem to be optimal values of instability (around $F=2 \times 10^{4}$ ) and stability (around $F=10^{4}$ ). The steady state at $F=10^{4}$ (Fig. 6a), plotted at criticality $\left(E_{c}=1.2 \times 10^{-7}\right)$ shows much more recirculation in the return flow compared to that in Fig. 3a. This is due to the much smaller Ekman number of the flow which makes it more inertially controlled. Although the pattern of the mode destabilizing this steady state derives (Fig. 6b-c) again from a westward propagating basin mode, its spatial scale has decreased substantially. It would be 'guessed' now to derive from the $(5,2)$ inviscid basin mode. Note that the critical Ekman number considered in the latter case is in the regime where numerical resolution was considered just sufficient to resolve steady states (Dijkstra and De Ruijter, 2000); these critical values may shift slightly when 
higher resolution is used.

\section{Results: Transient supercritical flows}

To investigate how the instabilities turn the steady states into transient flows as critical conditions are exceeded (i.e. values of $E$ smaller than those at the Hopf bifurcation), several transient flow computations are performed. The issues which are addressed in this section are the rectification of the mean flow due to the instabilities and the dominant frequency of variability in the nonlinear regime. Starting condition for all computations is an unstable steady state at the particular supercritical values of $E$ considered. Only the barotropic case is considered $\left(\mathrm{F}=10^{6}\right)$.

For $E=2.0 \times 10^{-7}$ and $H=1000 \mathrm{~m}$, the deviation from the perturbed steady state and the approach to the limit cycle can be seen in the retroflection index and, in equilibrium, regular periodic behavior results (Fig. 7). The period of the finite amplitude oscillation is slightly larger than one month and hence corresponds well to that of the eigenmode at the Hopf bifurcation in Fig. 2. When the flow patterns over one period of oscillation are considered and the difference of the instantaneous state and the unstable steady state (at $E=2.0 \times 10^{-7}$ ) is plotted, one actually sees the anomalies propagating as is suggested by the unstable mode at criticality (Fig. 3b-c). The time-averaged flow over exactly one oscillation period is shown in Fig. 7b together with the unstable steady state (Fig. 7c) at the same value of $E$. The differences between both flows are due to nonlinear rectification processes. The latter are small in this case, because the system is still not far above criticality $\left(E\right.$ near $\left.E_{c}\right)$. The retroflection index of the time mean state is $\mathcal{R}_{m}=-0.094$, whereas the steady state value is $\mathcal{R}_{s}=-0.014$ (Fig. 2). Hence, this indicates that the rectification due to barotropic instabilities decreases the degree of retroflection.

For the more inertially controlled case of $H=250 \mathrm{~m}$, the retroflection index varies 
so strongly that, at times, there is complete retroflection $\mathcal{R}=1$. The oscillations are irregular (Fig. 8) which indicates that several modes are involved in the dynamical behavior of the flow. Although the integration period is only two year, it is clear that the dominant frequency of the variability remains intermonthly, indicating that the first mode which is destabilized is still controlling the dynamics. The time mean state over the last year of the simulation for $E=2 \times 10^{-7}$ is shown in Fig. 8b, with the unstable steady state for the same value of $E$ shown in Fig. 8c. As can be seen, the steady state is substantially rectified, but the time-mean state still shares the same retroflection properties. The retroflection index of the time mean state is $\mathcal{R}_{m}=0.236$, whereas that of the steady state value is $\mathcal{R}_{s}=0.336$ (Fig. 2). Again, this indeed shows that the strongly time-dependent features of the flow due to barotropic instabilities decrease the degree of retroflection.

\section{Summary and Discussion}

In this paper, the stability of the steady flow states computed in a barotropic and reduced gravity shallow water model of the Agulhas Current system (Dijkstra and De Ruijter, 2000) for the idealized geometry of De Ruijter and Boudra (1985) was considered. All steady states destabilize at some critical value of the lateral friction (i.e. horizontal Ekman number $E_{c}$ ), which depends on the layer depth and the reduced gravity. In each case, the first mode which is destabilized has a pattern which is strongly related to a Rossby basin mode. Its period is not very dependent on the layer depth $\mathrm{H}$ (and thus on inertia), but strongly dependent on the value of the reduced gravity and varies from intermonthly in the barotropic case to interannual for the standard value of $g^{\prime}$ (i.e. $\mathrm{F}=10^{4}$ in Fig. 5).

For $E$ slightly smaller than $E_{c}$, periodic behavior appears. The destabilized basin modes are the origin of the variability on the steady state which consists of 'ring like' 
localized patterns propagating westwards around the continent. The frequency is determined by that of the first mode which destabilizes at criticality. Under slightly supercritical conditions, the time mean state of the time-dependent flow is different than the unstable steady state at the same values of the parameters through rectification processes. The retroflection of this time-mean state is, for the cases considered, smaller than that for the unstable steady state. This is an important result since it means that the actual retroflection in the time-mean state is inherited from the steady balances. Whether baroclinic instabilities and their rectification promote retroflection needs further study. For smaller values of $H$, the instabilities occur already on steady states with a weak retroflection. The transient flow is therefore more complicated at strong supercritical conditions, due to the destabilization of more modes (with respect to situations at larger $H$ ). However, the dominant period of the transient flow still appears to be in the intermonthly range and the time-mean state again shows a lower degree of retroflection than the steady state.

The results here indicate that the origin of the frequency of 'ring formation' is set by the physics of the large scale barotropic instabilities and hence provide a deeper level of understanding of those in Chassignet and Boudra (1988). One can now conclude that, in their case E1, the period of ring shedding indeed derives from a barotropic instability of a Rossby basin mode. This is fully consistent with the time scale found and the patterns of anomalies in the lower layer. In their upper layer, the Rossby basin mode signal is not so clear, likely because of strong interactions of the unstable mode with the upper layer flow. The barotropic instability mechanism here cannot explain the decrease in frequency in the inertial regime. Indeed, as Chassignet and Boudra (1988) mention, baroclinic processes are likely important for this to occur.

When the Rossby deformation radius is decreased (smaller $F$ ), or the wind stress increased at constant $F$, at some point the upper layer thickness will become zero somewhere in the domain. This limit may actually be interesting, since it probably 
forms the connection between results in Chassignet and Boudra (1988) and those in Pichevin et al. (1999). In Pichevin et al. (1999), the background flow structure is prescribed, through inflow/outflow conditions, without considering its origin in the wind and/or buoyancy forcing. In a near inertial flow in this configuration, retroflection is guaranteed to occur (Ou and De Ruijter, 1986) even with slip conditions at the continental boundaries. However, the coupling of the mean retroflecting current to the large scale wind forcing, and consequently to the far field flow, is important. Hence, central question becomes whether these types of background flows can be generated by a reasonable forcing.

Retroflection, and consequently ring formation, only exists under no-slip conditions in the set-up of Chassignet and Boudra (1988) as their model configuration does not allow upper interface outcrop. All flows are forced under a given wind stress, but one can argue that these flows may be are still too viscous to realize a near inertial current. Moreover, the effect of the lateral boundaries (in particular the closed western boundary) maybe too strong. Hence, here the question is whether a momentum imbalance is approached within a more global domain in the limit of very small lateral friction. If one can set-up the forcing of the wind and the basin geometry such that a type of retroflecting flow appears as in Pichevin et al. (1999), then the occurrence of a saddle node bifurcation would be the signature of the 'retroflection paradox' in the language of dynamical systems; work on this connection is currently is progress. 


\section{Acknowledgements}

This work was supported by the Netherlands Organization for Scientific Research (NWO) under a NWO-PIONIER grant to HD. WdR is supported by the Dutch National Research Program on Global Change (NRP II) under grant 013001237.10. All computations were performed on the CRAY C90 at the Academic Computing Centre (SARA), Amsterdam, the Netherlands within the project SC498. Use of these computing facilities was sponsored by the National Computing Facilities Foundation with financial support from NWO. 


\section{References}

Chassignet, E. P. and D. Boudra, 1988: Dynamics of the Agulhas retroflection and ring formation in a numerical model: II. Energetics and ring formation. J. Phys. Oceanography, 18, 304-319.

De Ruijter, W. and D. B. Boudra, 1985: The wind-driven circulation in the SouthAtlantic Indian Ocean. I. Numerical experiments in a one-layer model. Deep Sea Research, 32, 557-574.

De Ruijter, W. P. M., B. A., S. S. Drijfhout, J. R. E. Lutjeharms, R. P. Matano, T. Pichevin, P. J. van Leeuwen, and W. Weijer, 1999: Indian-Atlantic interocean exchange: dynamics, estimation and impact. J. Geophysical Res., 104, 20,885-20,910.

Dijkstra, H. A. and W. P. M. De Ruijter, 2000: On the physics of the Agulhas current: Steady retroflection regimes. J. Phys. Oceanography, xxx, submitted.

Dijkstra, H. A. and C. A. Katsman, 1997: Temporal variability of the wind-driven quasigeostrophic double gyre ocean circulation: Basic bifurcation diagrams. Geophys. Astrophys. Fluid Dyn., 85, 195-232.

Feron, R. C. V., W. P. M. De Ruijter, and D. Oskam, 1992: Ring-shedding in the Agulhas Current system. J. Geophysical Res., 97, 9467-9477.

Gordon, A. L., 1985: Indian - Atlantic transfer of thermocline water at the Agulhas Retroflection. Science, 227, 1030-1033.

Gordon, A. L. and W. F. Haxby, 1990: Agulhas eddies invade invade the South Atlantic: evidence from GEOSAT altimeter and shipboard CTD survey. J. Geophysical Res., 95, 3117-3125. 
Keller, H. B., 1977: Numerical solution of bifurcation and nonlinear eigenvalue problems. In Applications of Bifurcation Theory, Rabinowitz, P. H., editor. Academic Press, New York, U.S.A.

Nof, D. and T. Pichevin, 1996: The retroflection paradox. J. Phys. Oceanography, 26, 2344-2358.

Ou, H. W. and W. P. M. De Ruijter, 1986: Separation of an inertial boundary current from an irregular coastline. J. Phys. Oceanography, 16, 280-289.

Pedlosky, J., 1987: Geophysical Fluid Dynamics. Springer-Verlag, New York, U.S.A.

Pichevin, T., D. Nof, and J. R. E. Lutjeharms, 1999: Why are there Agulhas rings? J. Phys. Oceanography, 29, 693-707.

Schouten, M. W., W. P. M. de Ruijter, P. J. van Leeuwen, and J. R. E. Lutjeharms, 2000: Translation, decay and splitting of Agulhas rings in the southeastern Atlantic Ocean. J. Geophysical Res., 105, 21,913-21,925.

Seydel, R., 1994: Practical Bifurcation and Stability Analysis: from Equilibrium to Chaos. Springer-Verlag, New York, U.S.A.

Sleijpen, G. L. G. and H. A. Van der Vorst, 1996: A Jacobi-Davidson iteration method for linear eigenvalue problems. SIAM J. Matrix Anal. Appl., 17, 410-425.

Van Dorsselaer, J. J., 1997: Computing eigenvalues occurring in continuation methods with the Jacobi-Davidson QZ method. J. Comp. Physics, 138, 714-733. 


\section{Captions to the figures}

Fig. 1

Profile of the wind stress shape function $\tau^{\phi}$ in (1).

Fig. 2

Bifurcation diagrams for two barotropic cases with different layer depths $H$ computed with $1 / 4^{\circ}$ resolution. The first Hopf bifurcations, where the steady states become unstable to oscillatory instabilities, are marked on both branches with a black triangle. A drawn (dotted) linestyle indicates a stable (unstable) steady state.

Fig. 3

(a) Steady state at the Hopf bifurcation $\left(E_{c}=2.6 \times 10^{-7}\right)$ on the curve for $H=1000 \mathrm{~m}$ in Fig. 2, shown as a contour plot of the layer thickness anomaly $h$. (b) Real part of the eigenvector $\left(\hat{\mathbf{x}}_{R}\right)$ at the Hopf bifurcation. (c) Imaginary part of the eigenvector $\left(\hat{\mathbf{x}}_{I}\right)$ at the Hopf bifurcation. These patterns can be identified as a destabilized Rossby basin mode. In this case, the presence of the continent deforms the basin mode as it propagates westwards.

Fig. 4

(a) Steady state at the Hopf bifurcation $E_{c}=8.2 \times 10^{-7}$ for $H=250 \mathrm{~m}$ in Fig. 2, shown as a contour plot of the layer thickness anomaly $h$. (b) Real part of the eigenvector $\left(\hat{\mathbf{x}}_{R}\right)$ at the Hopf bifurcation. (c) Imaginary part of the eigenvector $\left(\hat{\mathbf{x}}_{I}\right)$ at the Hopf bifurcation. Together, $\hat{\mathbf{x}}_{R}$ and $\hat{\mathbf{x}}_{I}$ provide a picture of the propagating mode, according to $(11)$.

Fig. 5

Dependence of the critical Ekman number $E_{c}$ (drawn) at which the steady state becomes unstable to an oscillatory mode and the angular frequency $\left(\sigma_{i}\right)$ at criticality (dashed) on the Froude number $F$. The markers correspond to the actually computed points, which are connected by straight lines. 
Fig. 6

(a) Steady state at the Hopf bifurcation for $F=10^{4}, E_{c}=1.2 \times 10^{-7}$ and $H=1000$ m. (b) Real part of the eigenvector $\left(\hat{\mathbf{x}}_{I}\right)$ at the Hopf bifurcation. (c) Imaginary part of the eigenvector $\left(\hat{\mathbf{x}}_{R}\right)$ at the Hopf bifurcation.

Fig. 7

(a) Time series of the retroflection index for the barotropic case with $H=1000 \mathrm{~m}$, $E=2.0 \times 10^{-7}$. Starting point is the unstable steady state which is slightly perturbed into the direction of the mode destabilizing at the Hopf bifurcation. (b) Time mean state averaged over exactly one oscillation period in Fig. 7a. (c) The unstable steady state calculated at the same value of the Ekman number $\left(H=1000 \mathrm{~m}, E=2 \times 10^{-7}\right)$. Fig. 8

(a) Time series of the retroflection index for the barotropic case with $H=250 \mathrm{~m}$, $E=2.0 \times 10^{-7}$. (b) Time mean state computed over one year (over the interval as indicated by the dash-dotted line in Fig. 8a). (c) The unstable steady state at the same value of the Ekman number. 


\begin{tabular}{|c|c|c|c|}
\hline Dimensional parameters & \multicolumn{3}{|c|}{} \\
\hline Parameter & Value & Parameter & Value \\
\hline$r_{0}$ & $6.4 \times 10^{7} \mathrm{~m}$ & $\tau_{0}$ & $2.0 \times 10^{-1} \mathrm{~Pa}$ \\
\hline$H$ & $1.0 \times 10^{3} \mathrm{~m}$ & $A_{H}$ & $3.3 \times 10^{2} \mathrm{~m}^{2} \mathrm{~s}^{-1}$ \\
\hline$g^{\prime}$ & $0.1 \mathrm{~ms}^{-2}$ & $U$ & $1.0 \times 10^{-1} \mathrm{~ms}^{-1}$ \\
\hline$\rho_{0}$ & $1.0 \times 10^{3} \mathrm{kgm}^{-3}$ & $\Omega$ & $7.5 \times 10^{-5} \mathrm{~s}^{-1}$ \\
\hline Dimensionless parameters & \multicolumn{3}{|c}{} \\
\hline Parameter & Value & Parameter & Value \\
\hline$\alpha$ & $1.4 \times 10^{-3}$ & $E$ & $5.0 \times 10^{-8}$ \\
\hline$\epsilon$ & $1.0 \times 10^{-4}$ & $F$ & $1.0 \times 10^{4}$ \\
\hline
\end{tabular}

Table 1: Standard values of parameters in the (equivalent) barotropic model for the idealized small basin. The value of $A_{H}$ (and consequently $E$ ) is the reference value and $E$ will be used here as control parameter. 


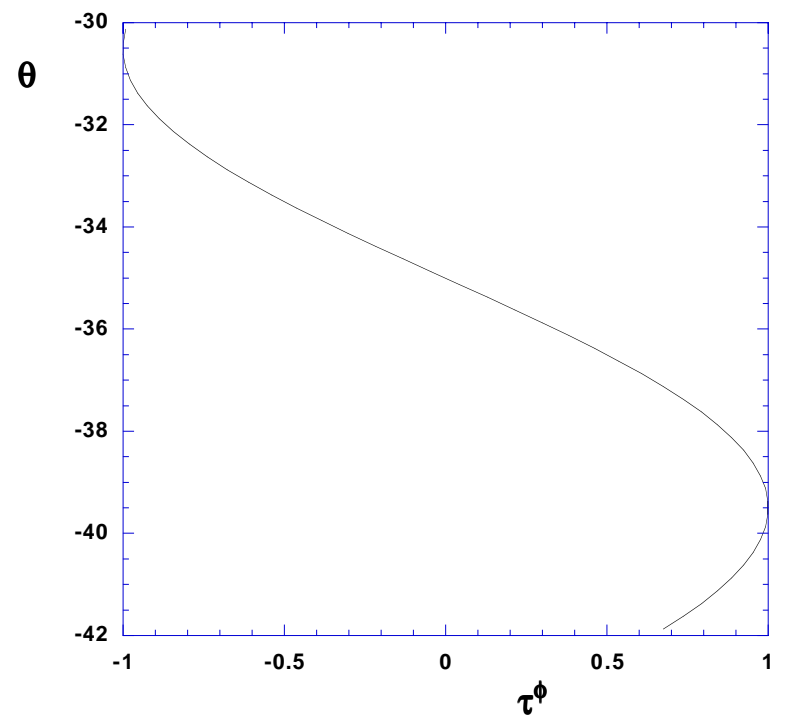

Figure 1: Profile of the wind stress shape function $\tau^{\phi}$ in (1). 


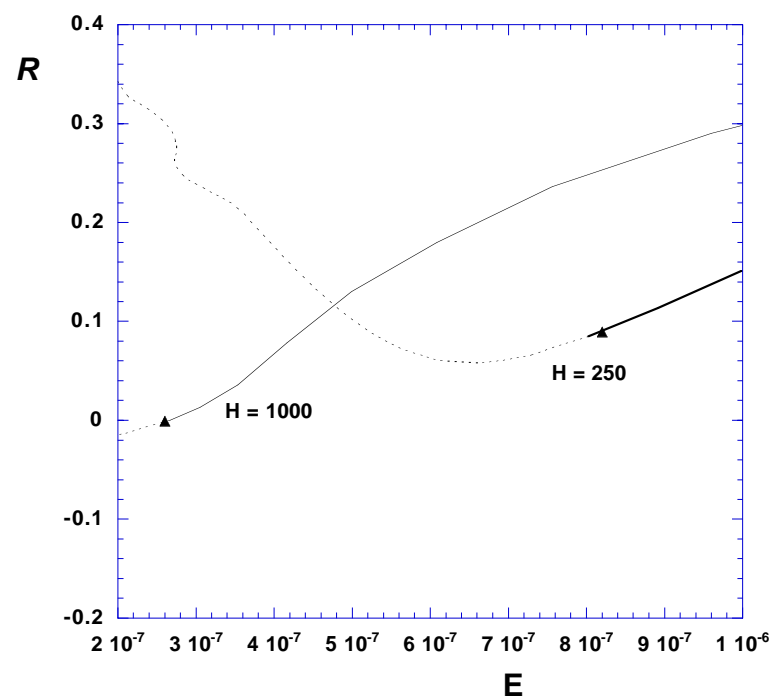

Figure 2: Bifurcation diagrams for two barotropic cases with different layer depths $H$ computed with $1 / 4^{\circ}$ resolution. The first Hopf bifurcations, where the steady states become unstable to oscillatory instabilities, are marked on both branches with a black triangle. A drawn (dotted) linestyle indicates a stable (unstable) steady state. 


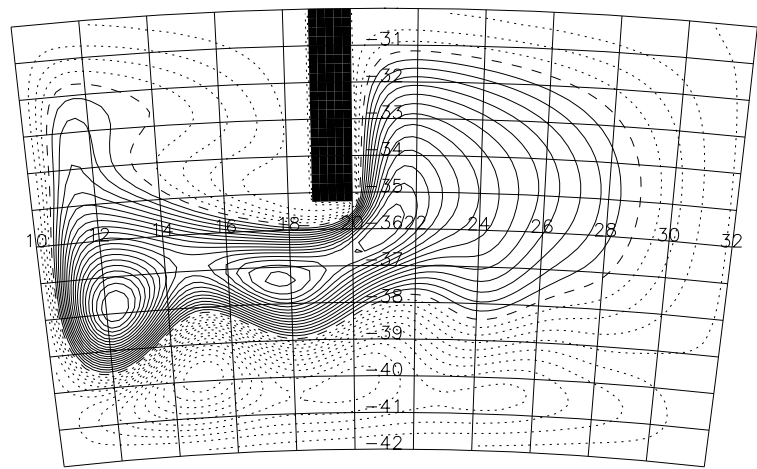

(a)

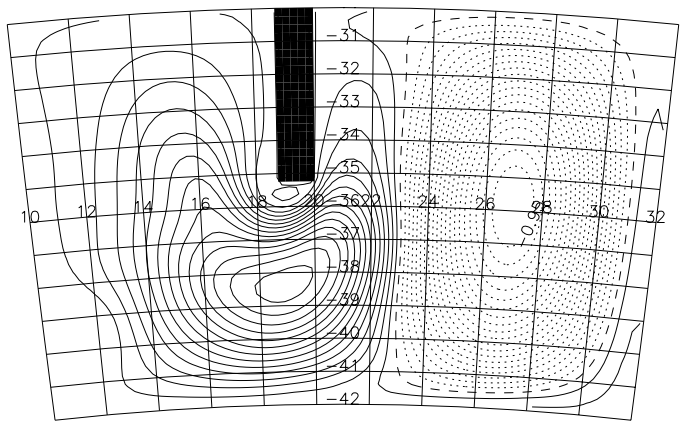

(b)

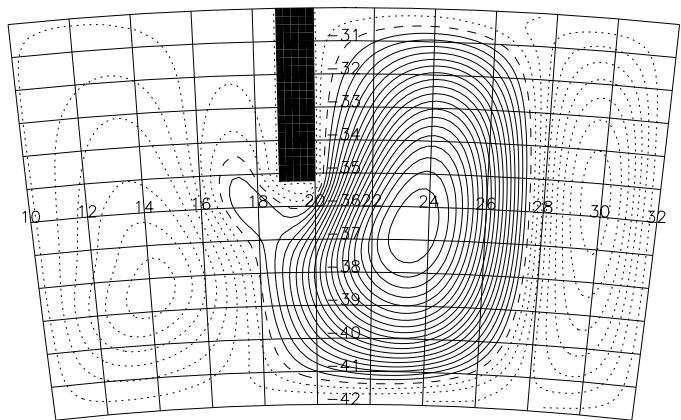

(c)

Figure 3: (a) Steady state at the Hopf bifurcation $\left(E_{c}=2.6 \times 10^{-7}\right)$ on the curve for $H=$ $1000 m$ in Fig. 2, shown as a contour plot of the layer thickness anomaly h. (b) Real part of the eigenvector $\left(\hat{\mathbf{x}}_{R}\right)$ at the Hopf bifurcation. (c) Imaginary part of the eigenvector $\left(\hat{\mathbf{x}}_{I}\right)$ at the Hopf bifurcation. These patterns can be identified as a destabilized Rossby basin mode. In this case, the presence of the continent deforms the basin mode as it propagates westwards. 


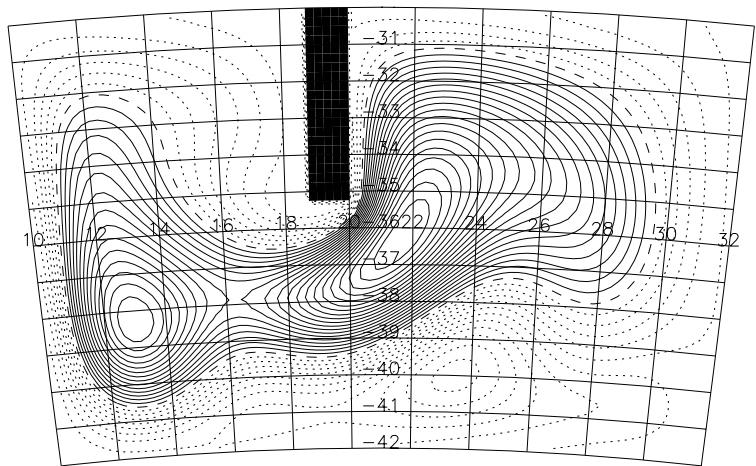

(a)

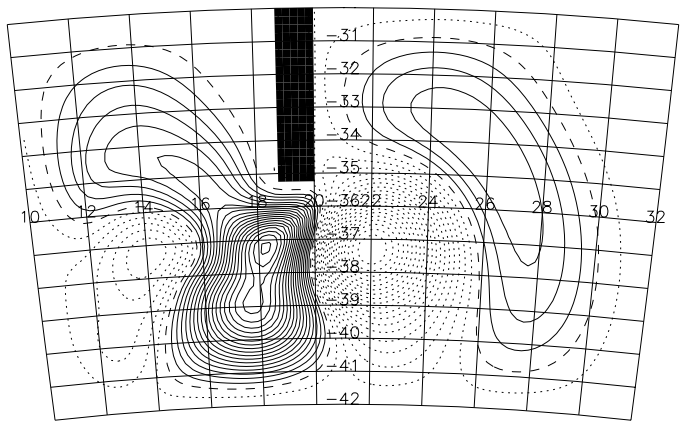

(b)

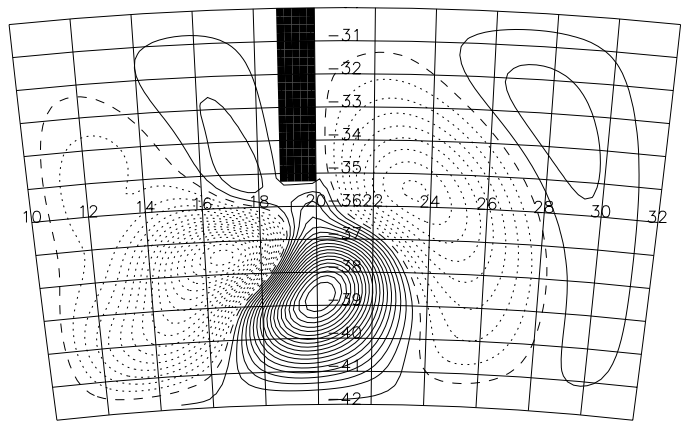

(c)

Figure 4: (a) Steady state at the Hopf bifurcation $E_{c}=8.2 \times 10^{-7}$ for $H=250 \mathrm{~m}$ in Fig. 2, shown as a contour plot of the layer thickness anomaly $h$. (b) Real part of the eigenvector $\left(\hat{\mathbf{x}}_{R}\right)$ at the Hopf bifurcation. (c) Imaginary part of the eigenvector $\left(\hat{\mathbf{x}}_{I}\right)$ at the Hopf bifurcation. Together, $\hat{\mathbf{x}}_{R}$ and $\hat{\mathbf{x}}_{I}$ provide a picture of the propagating mode, according to (11). 


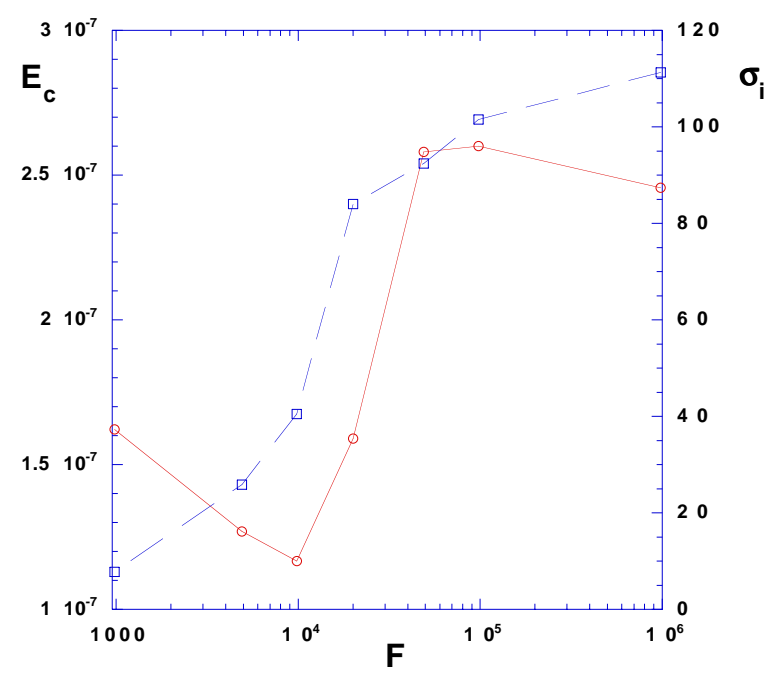

Figure 5: Dependence of the critical Ekman number $E_{c}$ (drawn) at which the steady state becomes unstable to an oscillatory mode and the angular frequency $\left(\sigma_{i}\right)$ at criticality (dashed) on the Froude number $F$. The markers correspond to the actually computed points, which are connected by straight lines. 


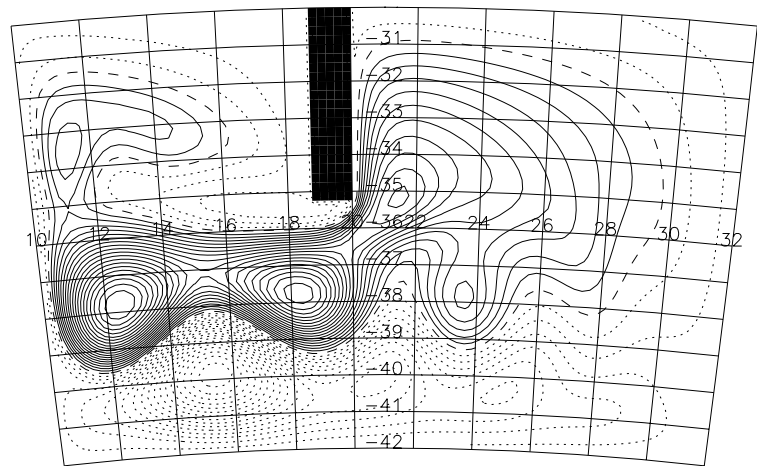

(a)

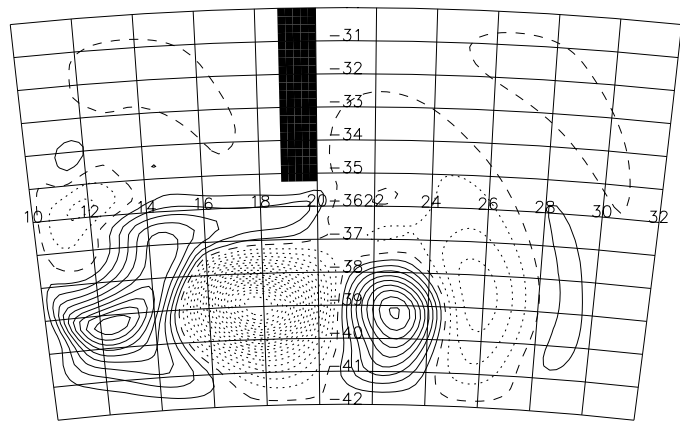

(b)

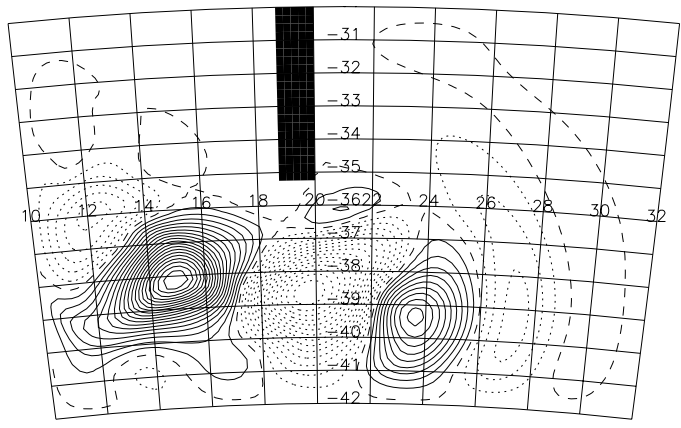

(c)

Figure 6: (a) Steady state at the Hopf bifurcation for $F=10^{4}, E_{c}=1.2 \times 10^{-7}$ and $H=1000$ m. (b) Real part of the eigenvector $\left(\hat{\mathbf{x}}_{I}\right)$ at the Hopf bifurcation. (c) Imaginary part of the eigenvector $\left(\hat{\mathbf{x}}_{R}\right)$ at the Hopf bifurcation. 

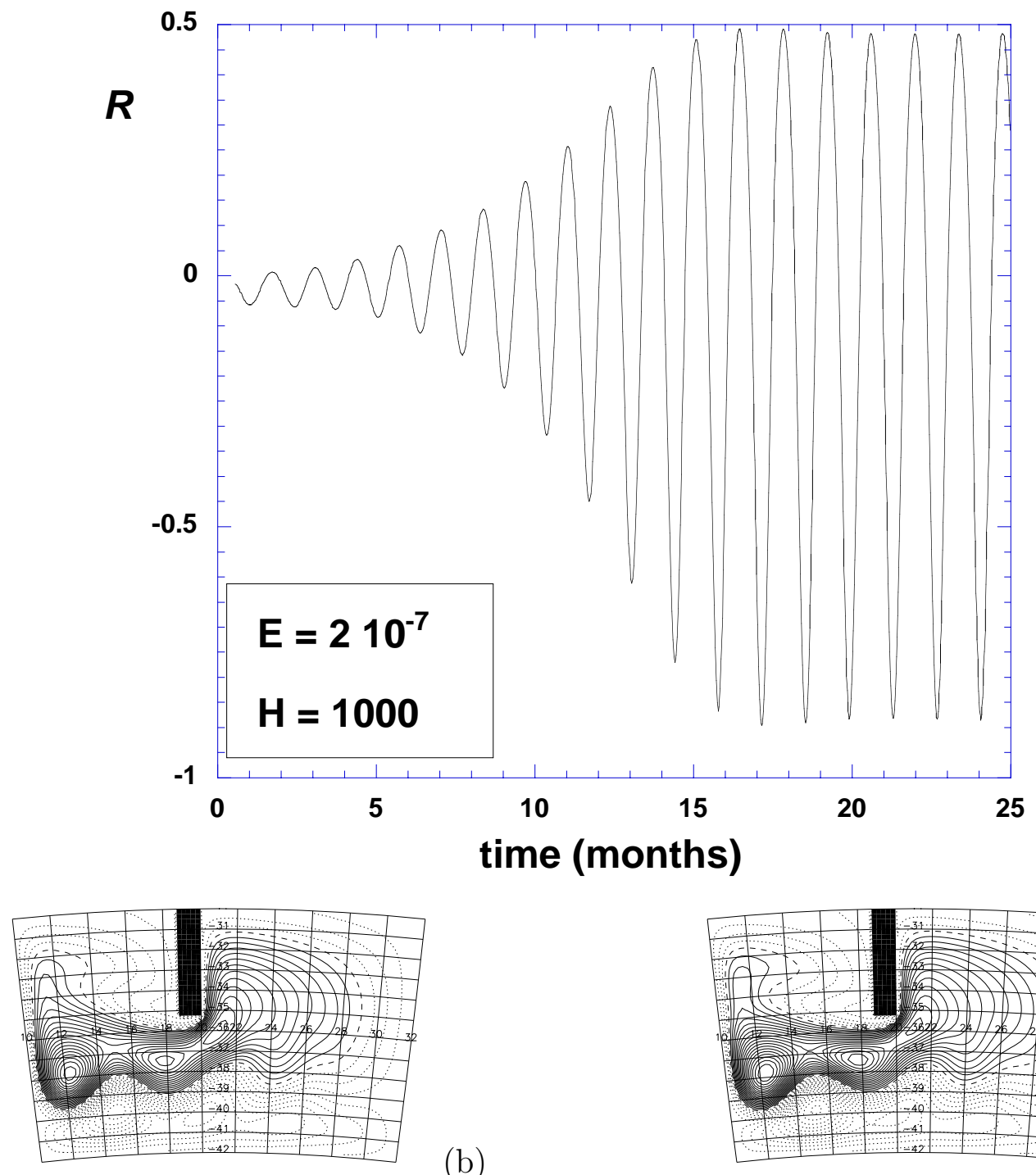

(b)

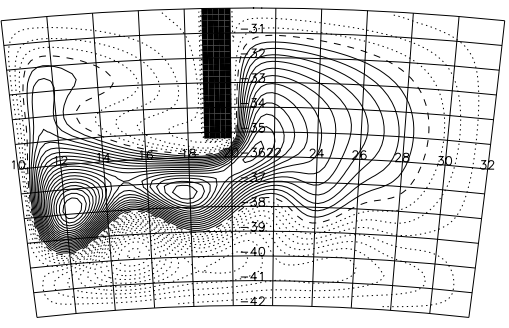

(c)

Figure 7: (a) Time series of the retroflection index for the barotropic case with $H=1000 \mathrm{~m}$, $E=2.0 \times 10^{-7}$. Starting point is the unstable steady state which is slightly perturbed into the direction of the mode destabilizing at the Hopf bifurcation. (b) Time mean state averaged over exactly one oscillation period in Fig. 7a. (c) The unstable steady state calculated at the same value of the Ekman number $\left(H=1000 m, E=2 \times 10^{-7}\right)$. 

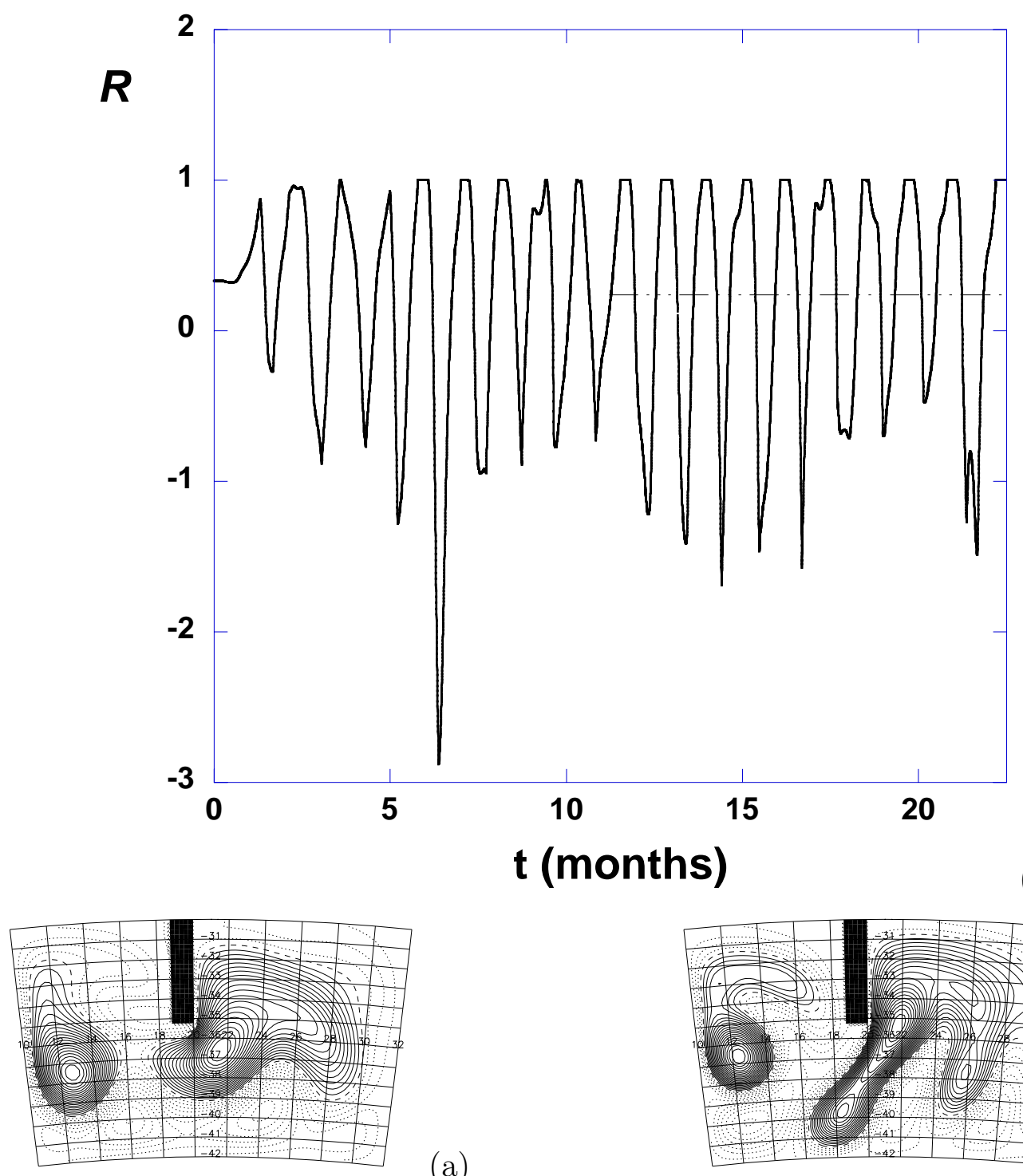

(a)

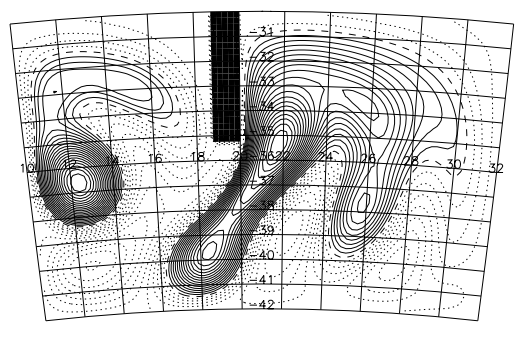

(b)

Figure 8: (a) Time series of the retroflection index for the barotropic case with $H=250 \mathrm{~m}$, $E=2.0 \times 10^{-7}$. (b) Time mean state computed over one year (over the interval as indicated by the dash-dotted line in Fig. 8a). (c) The unstable steady state at the same value of the Ekman number. 